\title{
THE INFLUENCE OF INTRA- ABDOMINAL HYPERTENSION ON THE CENTRAL NERVOUS SYSTEM: CURRENT INSIGHTS AND CLINICAL RECOMMENDATIONS: IS IT ALL IN THE HEAD?
}

\author{
I. De laet ${ }^{1}$, G. Citerio², M.L.N.G. Malbrain ${ }^{1}$
}

\begin{abstract}
Introduction: Intra-abdominal hypertension (IAH) and abdominal compartment syndrome are a common occurrence in ICU patients. The deleterious effects of IAH on organ function are well known and increasingly appreciated in recent years, especially where renal and respiratory function are concerned.

Methods: This review will focus on the available literature from the last years. A Medline and PubMed search was performed in order to find an answer to the question "What is the impact of increased IAP on neurologic function in the critically ill?"
\end{abstract}

\footnotetext{
ZiekenhuisNetwerk Antwerpen,

Campus Stuivenberg,

Intensive Care Unit,

Antwerp, Belgium;

2 Hospital San Gerardo,

UO Neuroanesthesia e Neurorianimazione,

Dipartimento di Mzedicina Perioperatoria e Terapie Intensive, Monza, Italy
}

Address for correspondence:

Manu Malbrain, MD

ICU Director

ZiekenhuisNetwerk Antwerpen

Campus Stuivenberg

Lange Beeldekensstraat 267

B-2060 Antwerpen 6

Belgium

Tel: +32 32177399

Fax: +3232177574

E-mail: manu.malbrain@skynet.be
Results: The amount of data on the influence of IAH on the central nervous system is more scarce, but several animal and human studies have demonstrated a clear correlation between intraabdominal pressure (IAP) and intracranial pressure (ICP). This correlation is probably due to transmission of the increased IAP to the thorax leading to increased intrathoracic, pleural pressure and central venous pressure, decreased venous return from the brain and increased ICP. This hypothesis is supported by the observation that the increase in ICP is abolished when a sternotomy and pleuropericardotomy are performed, and by the fact that abdominal decompression has produced good results in treating refractory intracranial hypertension $(\mathrm{ICH})$ in patients with both $\mathrm{IAH}$ and $\mathrm{ICH}$.

Conclusions: A close relationship between IAP and ICP has been observed in several animal and human studies. The clinical impact of this association is dependent on the baseline ICP and the compensatory reserve of the patient. Some studies have reported good results in treating refractory $\mathrm{ICH}$ by abdominal decompression in patients with concomitant IAH. Monitoring of IAP and ICP in risk patients is essential.

\section{INTRODUCTION}

Intra-abdominal hypertension (IAH) and abdominal compartment syndrome (ACS) are a relatively common occurrence in ICU patients as was demonstrated by Malbrain et al $(1,2)$. Interest in this topic has increased dramatically over the last few years leading to a rap- 
idly growing body of evidence regarding all aspects of IAH. The deleterious effect of IAH on organ function has been well documented $(3,4)$. Especially the organs lying within or adjacent to the abdominal cavity (e.g. the kidney) are at risk due to decreased blood supply and direct pressure on the organ involved (5). However, also distant organs, such as the heart and the brain, are influenced by IAH in ways that are not yet completely understood $(6,7)$. Several animal studies and human clinical studies have described the relationship between intra-abdominal pressure (IAP) and intracranial pressure (ICP) (8-13) and some have offered insights into the mechanisms involved $(7,14-19)$. Some small series describing successful treatment for patients with IAH and intracranial hypertension $(\mathrm{ICH})$ have also been published $(10,20)$.

In this review we will summarize all research findings relevant to the subject, offer an overview of current knowledge on this fascinating topic and make a few clinical recommendations based on the findings described.

\section{THE RELATIONSHIP BETWEEN IAP AND ICP}

\section{Animal studies}

The first report describing a relationship between IAP and ICP was published in 1994 by Josephs et al of the Boston University School of Medicine who induced $\mathrm{IAH}$ in a porcine model (by installing a pneumoperitoneum) and observed a concomitant increase in ICP but no significant decrease in cerebral perfusion pressure (CPP) (11). The same effect could be observed when the added insult of brain injury (by inflating a balloon in the epidural space) was induced. The authors explained this phenomenon by formulating a hypothesis based on the modified Monro-Kellie doctrine. The Monro-Kellie doctrine recognizes three main contents in the cranial space: vascular, cerebrospinal fluid (CSF) and parenchyma. The doctrine states that, in adults, changes in one or more of these contents result in reciprocal changes in the remaining compartments. The imbalance of the contents produces an ICP increase when buffer mechanisms are exhausted. In other words, ICP reflects the relationship between the volume of intracranial contents (vascular, CSF, and parenchyma) and the volume of the cranial vault. In their brain injury model, Josephs et al. increased the intracranial volume directly by inflating an epidural balloon (11). At the same time they indirectly increased the vascular contents by inducing a pneumoperitoneum which decreases thoraco-abdomi- nal compliance and reduces venous outflow from the brain, thus producing an ICP rise.

The confirmation of Josephs' hypothesis came a few years later. Using a porcine model of acutely elevated IAP, Bloomfield et al. clarified the mechanisms by which IAH increases ICP and decreases CPP, evaluated the effect of intravascular volume expansion upon ICP and CPP and studied the relationship between IAP, pleural pressure (PP), CVP and ICP $(8,9)$. They measured the effects of elevated IAP upon ICP and CPP before and after intravascular volume resuscitation (8). IAP was increased in 5 anesthetized swine by inflating an intraperitoneal balloon until $25 \mathrm{mmHg}$ above baseline. Intravascular volume was then expanded and, finally, abdominal decompression was performed.

Bloomfield demonstrated that elevated IAP increases CVP, PP, ICP and decreases cardiac index (CI), mean arterial pressure (MAP) and CPP. Moreover, volume expansion, in the presence of elevated IAP, further raised the ICP and CPP (because of a larger increase in MAP). An interesting finding was that an IAP greater than or equal to $25 \mathrm{mmHg}$ produced a statistically significant decrease in CPP, even in animals without head injuries.

The results of this study confirmed that the mechanism of increment of ICP is mechanical. In accordance with the Monro-Kellie doctrine, the authors demonstrated that cerebral venous outflow via the jugular venous system was impeded by a significant rise in the CVP. This phenomenon was amplified by volume expansion, due to the additional rise in CVP. The authors suggested that the functional obstruction of the jugular venous system and the ensuing obligatory increase in the volume of the cerebral vascular space, are the mechanisms responsible for the increased ICP caused by elevated IAP.

One year later, the same group published a new study with a similar setup, only this time the pigs were divided into two groups (9). The first group was treated as mentioned before, the second group underwent sternotomy and pleuropericardotomy before increasing IAP. Interestingly, in the second group, sternotomy and pleuropericardotomy abolished all effects of increased IAP, except the decrease in $\mathrm{Cl}$, which supports the hypothesis formulated in the first study (Figure 1).

In a similar study in 5 swine, Rosin et al. found no significant change from baseline in ICP when the abdominal pressure was $5 \mathrm{~mm} \mathrm{Hg}$ (21). However, ICP and CPP were affected by increasing the abdominal pressure to 15 and $25 \mathrm{~mm} \mathrm{Hg}(\mathrm{P}=0.035$ and 0.04 for ICP respectively). They concluded that low-pressure laparos- 
Panel A

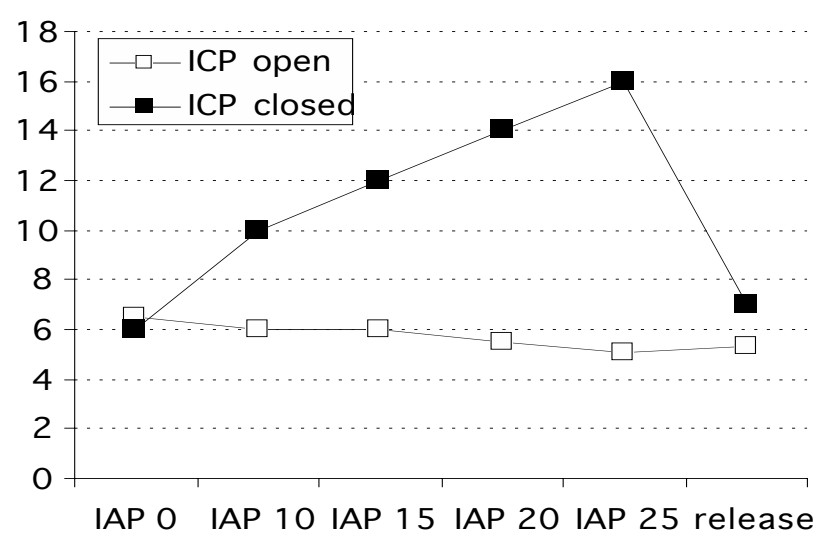

Panel B

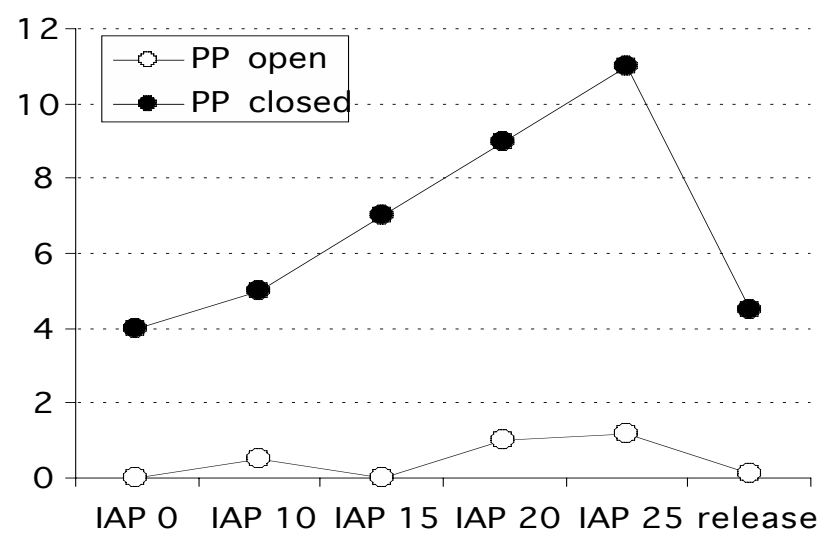

Figure 1:

Panel A: Effect of increasing intra-abdominal pressure (IAP) in closed and open-chest animals on ICP (ICP: filled boxes closed chest, open boxes animals with thoracotomy)

Panel B: Effect of increasing intra-abdominal pressure (IAP) in closed and open-chest animals on pleural pressure (PP: closed circles animals without sternotomy, open circles animals with sternotomy). Modified from Bloomfield (9)

copy may reduce the adverse effects of pneumoperitoneum on ICP and CPP. It may therefore be advisable to use low pressures in laparoscopic surgery, especially when changes in ICP or renal perfusion may have significant clinical implications $(21,22)$.

Hanel et al. measured cerebral hemodynamics in 10 pigs during laparoscopic insufflation to $12 \mathrm{mmHg}$ (23). Pneumoperitoneum significantly $(p<0.05)$ increased CVP from $6.3 \pm 2.1$ to $11.1 \pm 3.0 \mathrm{~mm} \mathrm{Hg}$ and sagittal sinus pressure from $8.0 \pm 2.8$ to $11.9 \pm 3.0 \mathrm{~mm} \mathrm{Hg}$ ). However, bilateral internal carotid artery blood flow ( $46.0 \pm 7.4 \mathrm{vs} 47.7 \pm 7.1 \mathrm{~mL} / 100 \mathrm{~g}$ per minute), cortical cerebral blood flow, CBF $(263 \pm 115$ vs $259 \pm 158$ tissue perfusion units), and subcortical CBF (131 \pm 145 vs 133 \pm 149 tissue perfusion units) did not change during $\mathrm{CO} 2$ pneumoperitoneum. The increases in sagittal sinus pressure are likely related to decreases in cerebral venous drainage caused by increases in IAP.

Other studies looked at the effect of combined abdominal and thoracic binding (ATB) on neurologic function during CPR: mean ICP was higher during ATB (46 \pm $2 \mathrm{~mm} \mathrm{Hg}$ ) than conventional CPR (20 $2 \mathrm{~mm} \mathrm{Hg})(24)$. However, the net brain perfusion pressure gradient (carotid artery pressure - ICP) was greater with ATB (14 $\pm 3 \mathrm{~mm} \mathrm{Hg})$ than with conventional CPR $(5 \pm 0.4 \mathrm{~mm}$ $\mathrm{Hg}$ ). Cerebral blood flow was significantly greater during ATB CPR ( $32 \pm 7 \%$ of prearrest cerebral flow) than during conventional CPR $(3 \pm 2 \%)$. They conclude that ATB CPR substantially improved brain perfusion by enhancing CPP in this experimental dog model. The same results were found by Ratjen in lambs (25).

Palafox looked at the effect of MAST suit on ICP in dogs (26). During MAST inflation, they found a rise in ICP that mirrored the rise in CVP and never reached potentially harmful levels in the hemorrhaged animals and animals with a mass intracranial lesion. In the animals with cardiac tamponade and tension pneumothorax, inflation of the abdominal portion of the MAST suit produced a marked rise in CVP and ICP. This was altered by either relieving the lesion or reducing the pressure of the abdominal portion of the MAST suit.

Schob compared the pathophysiological neurologic effects of carbon dioxide, helium and nitrous oxide pneumoperitoneum in 24 pigs (27). The mean ICP increased significantly in all groups during peritoneal insufflation compared with the control group $(P<$ $0.005)$. The CO2-insufflated animals also showed a significant increase in PaCO2 $(P<0.05)$ and ETCO2 $(P$ $<0.05)$, as well as a decrease in $\mathrm{pH}(\mathrm{P}<0.05)$. After inflating the epidural balloon the ICP remained significantly higher in animals inflated with $\mathrm{CO} 2$ as compared with the He and N2O groups $(P<0.05)$. They concluded that peritoneal insufflation with $\mathrm{He}$ and N2O resulted in a significantly less increase in ICP as compared with $\mathrm{CO}$. That difference was most likely due to a metabolically mediated increase in CPP. Further studies need to be conducted to determine the safety and efficacy of using He and N2O as inflation agents prior to attempting diagnostic or therapeutic laparoscopy in patients with potential closed head injuries. 
In conclusion, these laboratory studies clearly showed that an IAP rise decreases the thoracic-abdominal compliance and increases intrathoracic pressures. This in turn reduces cerebral venous outflow, causing an ICP elevation.

\section{Clinical studies}

Despite the convincing results of the previously mentioned animal studies, interest in this subject in human clinical research is very recent. In 1995, two early case reports were published that comfirmed the results of the animal studies (28-30), but the first well conducted clinical study evaluating the relationship between IAP and ICP was published by Citerio et al in 2001 (15). They conducted a prospective non-randomised observational study to systematically measure the effect of increased IAP in 15 patients with traumatic brain injury. IAP was increased by positioning a soft 15-L bag of water on the patient's abdomen. Naturally, patients could only be included in this study after the acute phase of their injury, when no intracranial hypertension was present. All patients were intubated and mechanically ventilated. Many parameters were measured: IAP, MAP, CVP, ICP, CPP, jugular bulb pressure (IJP), jugular bulb oxygen saturation and compliance of the respiratory system, divided into pulmonary and chest wall components. The authors found that placing a weight on the patient's abdomen generated a significant increase in IAP and a concomitant and rapid increase in CVP, IJP and ICP. All these changes required only seconds to reach a plateau and remained stably increased until the IAP returned to baseline after the weight removal (Figure 2).

Curiously, in this study a rise in MAP was observed, allowing for maintenance of a constant CPP in spite of increased ICP. This is not in concordance with the animal studies. This may be due to the lower levels of IAP generated in this human study compared to the animal studies and the use of different sedatives in animal and human studies or it may represent a true physiologic mechanism where increased intrathoracic pressure facilitates systolic ejection (although venous return is decreased). A second interesting finding from this study is that respiratory system compliance decreased significantly in all patients and this decrease was exclusively due to decreased compliance of the thoracic wall. Lung compliance did not change at all.

These findings confirm the hypothesis that IAH displaces the diaphragm upward, reducing the chest wall compliance and therefore respiratory system compliance. The pressure in the abdominal compartment (IAP) is directly transmitted to the thoracic compartment, where it increases intrathoracic pressures (CVP, esophageal pressure) and, thereafter to the cerebral compartment (causing an increase in IJP and ICP). In other words, the ICP rise appears to be the result of an obstruction to the cerebral venous drainage, causing elevation of pressure in the intracranial compartment.

Deeren et al found similar results in 11 patients with nontraumatic brain injury (20). They found tight associations between IAP and ICP, $\triangle \mathrm{I} A \mathrm{P}$ and $\triangle \mathrm{ICP}$ and $\triangle \mathrm{IAP}$ and $\triangle C P P$ even at only slightly increased levels of IAP (Figure 4). In total eleven patients were studied with simultaneous IAP and ICP-monitoring because of ischemic (in four), hemorrhagic (in five) and metabolic

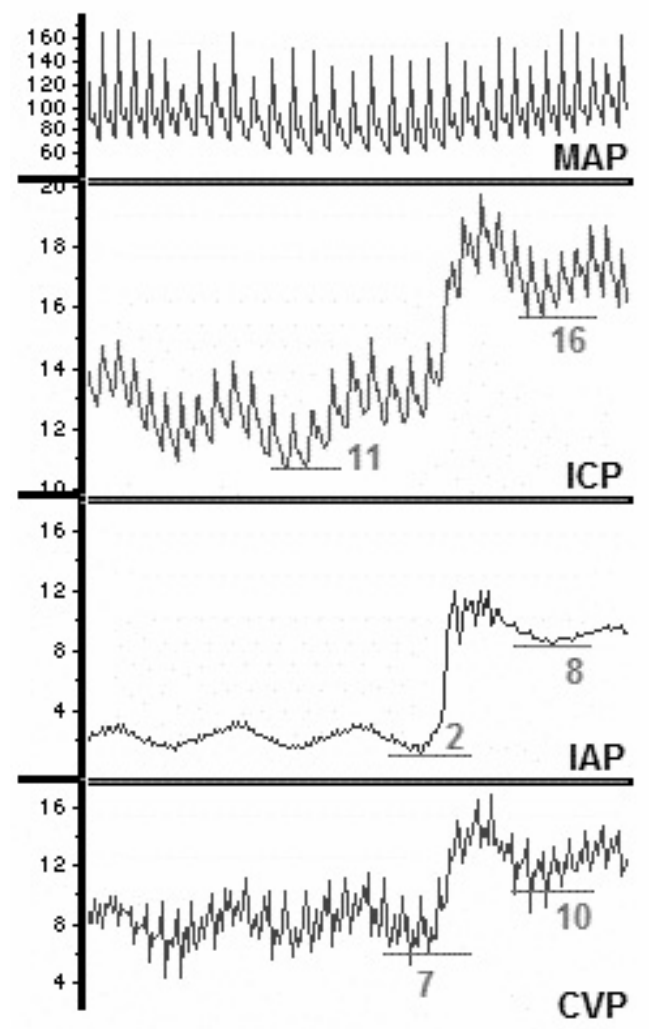

Figure 2: Effect of increased IAP, obtained with a weight application

- in a single patient. Computerized tracings are from top to bottom: MAP: mean arterial pressure; ICP: intracranial pressure; IAP: intra-abdominal pressure; CVP: central venous pressure. The IAP increased from 2 to $8 \mathrm{mmHg}$, this resulted in an increase in CVP from 7 to $10 \mathrm{mmHg}$ and an increase in ICP from 11 to $16 \mathrm{mmHg}$. Hence the transmission from the abdomen to the thorax compartment can be calculated as $\triangle C V P(=10-7)$ divided by $\triangle I A P(=8-2)$ or thus $50 \%$, and the transmission from the abdomen to the cranial compartment can be calculated as $\triangle / C P(=16$ - 11) divided by $\triangle I A P(=8-2)$ or thus $83 \%$. Modified from Citerio $(7,15)$ 


\section{Panel A}

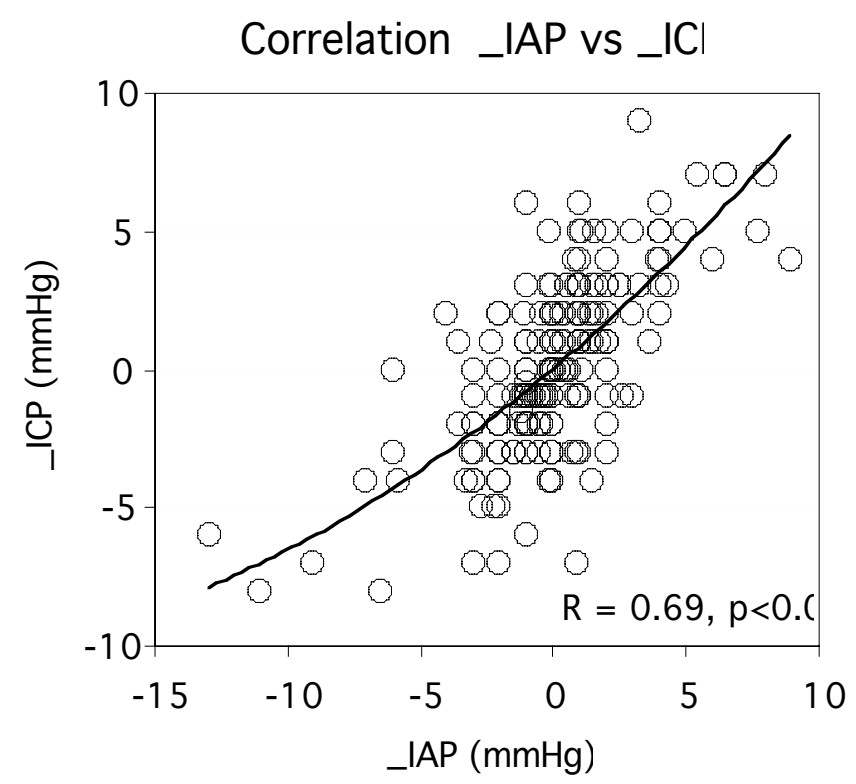

\section{Panel B}

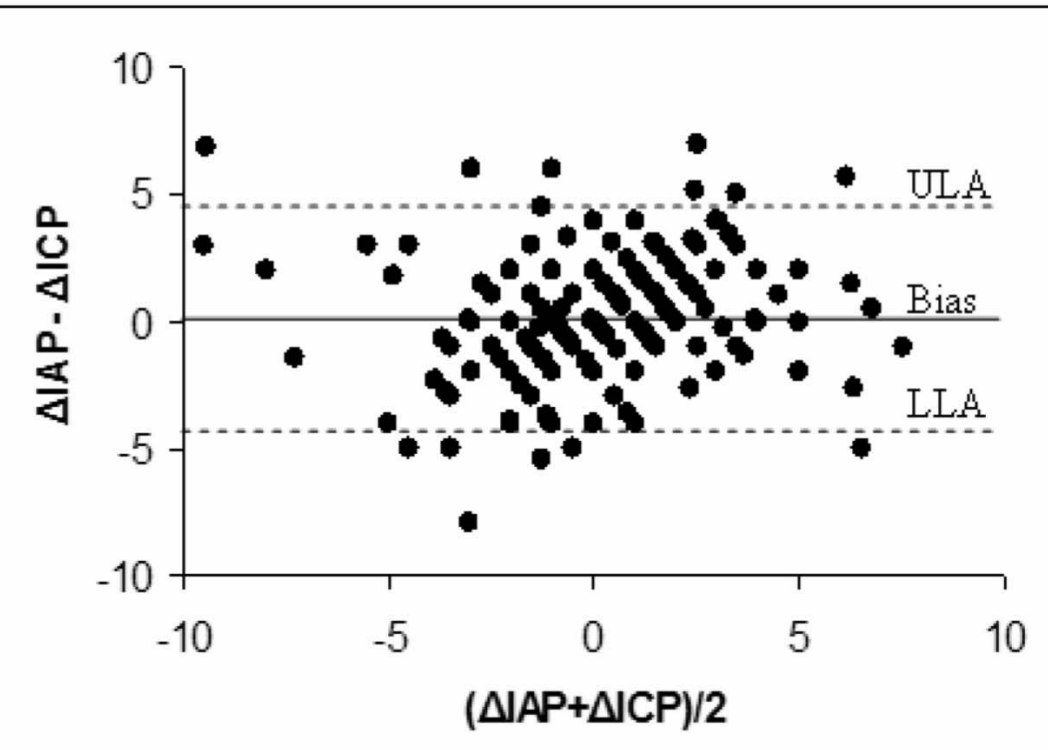

Figure 4: Panel A: regression analysis between $\triangle I A P$ versus $\triangle / C P$ (positive correlation), Panel B: Bland-Altman plot of $\triangle / A P$ and $\triangle / C P$

(ULA: upper limit of agreement, LLA: lower limit of agreement, IAP: intraabdominal pressure, ICP: intracranial pressure) (in two) encephalopathy. Two hundred fourteen consecutive paired data sets were compared. The mean IAP of each patient ranged from 3.8 to 11.8 $\mathrm{mmHg}$, with a mean ICP from 6.7 to $15 \mathrm{mmHg}$ and mean CPP from 70.8 to $123 \mathrm{mmHg}$. For ICP, the regression coefficient associated with IAP was 0.64 (standard error: 0.05 ; 95\% confidence interval: 0.56 to 0.73 ; $p$ 0.001 , partial correlation 0.70 ). For $\mathrm{CPP}$, the regression coefficient associated with IAP was -1.36 (standard error: $0.3 ; 95 \%$ confidence interval: 1.94 to -0.78 ; $p<0.001$; partial correlation -0.30 ).

Cooke investigated key symptoms and signs of raised ICP in 39 patients after laparoscopic abdominal surgery and compared them with a control group of 33 patients after open operations (31). They found that the incidence of headache and nausea was significantly higher in the laparoscopic group than in the control subjects and they concluded that these results could be explained by raised intracranial pressure exacerbated by the $\mathrm{CO} 2$ pneumoperitoneum, and that this effect is not mediated by raised expiratory $\mathrm{CO} 2$ levels intraoperatively.

Recently several reports have been published alluding to neurologic deterioration related to increased IAP in patients with ventriculoperitoneal shunt (VPS) dysfunction (32-34). These reports provide anecdotal evidence that transient and easily reversible increases in the IAP of adults with VPS can result in dysfunction. Although it may not be practical to delay shunt revision while attempting to correct constipation, meteorism, ileus, or small bowel obstruction, clinicians treating patients with these abdominal conditions should be aware that they could cause transient VPS failure.

Several reports also pointed towards obesity as a cause of increased 
IAP leading to idiopathic ICH and pseudotumor cerebri (35-38). Interestingly, negative abdominal pressure, weight loss and bariatric surgery have been demonstrated to reduce the clinical signs of ICH (headache, blurred vision,...) and ICP (39-43). Furthermore recent weight gain may be associated with relapses of idiopathic ICH (44). Obesity associated sleep apnea may also be associated with idiopathic ICH $(45,46)$.

\section{CLINICAL IMPORTANCE OF IAH IN PATIENTS AT RISK FOR ICH}

According to the previously mentioned modified Monro-Kellie doctrine, ICP remains constant over a relatively wide range of intracranial volume. In this plateau phase, an increase in intracranial volume due to e.g. cerebral edema, hematoma or mass lesion, will be compensated by the evacuation of cerebrospinal fluid through the foramen magnum at the base of the skull. However, when these compensation mechanisms are exhausted at higher intracranial volumes, any small increase in volume produces a marked increase in ICP rapidly leading to $\mathrm{ICH}$ (Figure 3 ).

When we combine this concept with the findings of the previously mentioned experimental and clinical studies we can speculate that in the presence of $\mathrm{ICH}$ the effect of increased IAP probably induces a more profound and harmful increase in ICP due to a different starting ICP position on the intracranial PV curve and different strain on the compensatory capacity. Therefore, it is crucial to measure IAP in patients with ICH at risk for IAH (or vice versa).

These findings are of great importance for patient care, for example in trauma patients, since abdominal

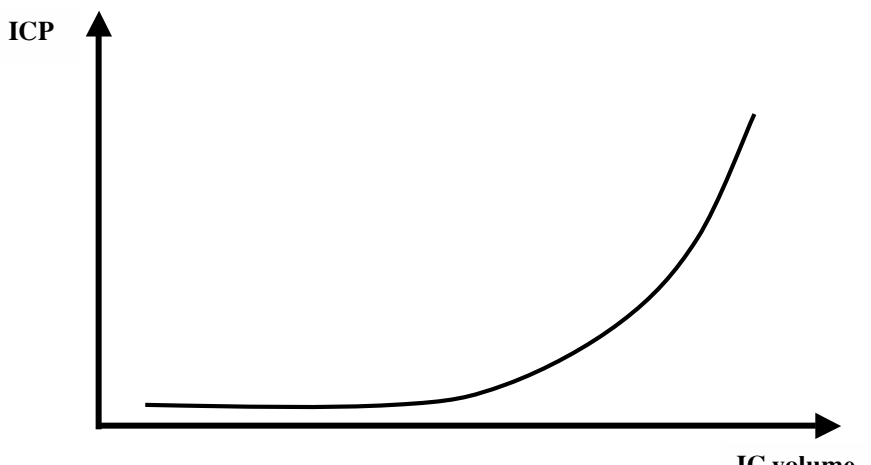

Figure 3: Relationship between intracranial volume (IC volume) and intracranial pressure (ICP) according to the MonroKellie doctrine trauma is commonly associated with head trauma and therefore patients with combined $\mathrm{ICH}$ and IAH may be more common in the ICU population than previously thought. Table 1 summarizes the neurologic effects of increased IAP.

\section{TREATMENT OPTIONS}

A few studies have addressed the issue of treatment of $\mathrm{ICH}$ in the presence of $\mathrm{IAH}$. A group of the Adams Cowley Shock Trauma Center of Maryland published a paper on the use of decompressive laparotomy for the treatment of intractable ICH after traumatic brain injury (10). This technique was used in 17 patients whose intracranial hypertension was unresponsive to maximal therapy. The decompression resulted in a marked decrease in both ICP and IAP. In six patients the decrease was transient and all patients died. The remaining 11 patients showed a persistent decrease in ICP and IAP and survived. The same remarks were posted in a letter by Lyons (47). Bloomfield also described the successful management of a patient with severe multisystem injury in whom abdominal decompression dramatically reduced high ICP unresponsive to medical measures (30).

Deeren et al published a clinical study in 2005 describing the relationship between IAP and ICP in patients with nontraumatic brain injury (Figure 5). In four of these patients IAP and ICP measurements were obtained before and after administration of a neuromuscular blocker (20). Both IAP and ICP decreased and CPP and abdominal perfusion pressure (APP) decreased significantly after administration of a 10mg bolus of cisatracurium. Both IAP and ICP also dropped towards levels

\section{Table 1}

\begin{tabular}{l}
\hline Neurologic effects related to IAP \\
\hline Intracranial pressure $\uparrow$ \\
Cerebral perfusion pressure $\downarrow$ \\
Cerebral blood flow $\downarrow$ \\
Jugular bulb saturation $\downarrow$ \\
Cerebral venous outflow $\downarrow$ \\
Cerebrovascular resistance $\uparrow$ \\
Idiopathic intracranial hypertension in morbid obesity \\
Pseudotumor cerebri in morbid obesity
\end{tabular}

Neurologic effects reversed after bariatric surgery or weight loss Neurologic deterioration during laparoscopy 
Panel A
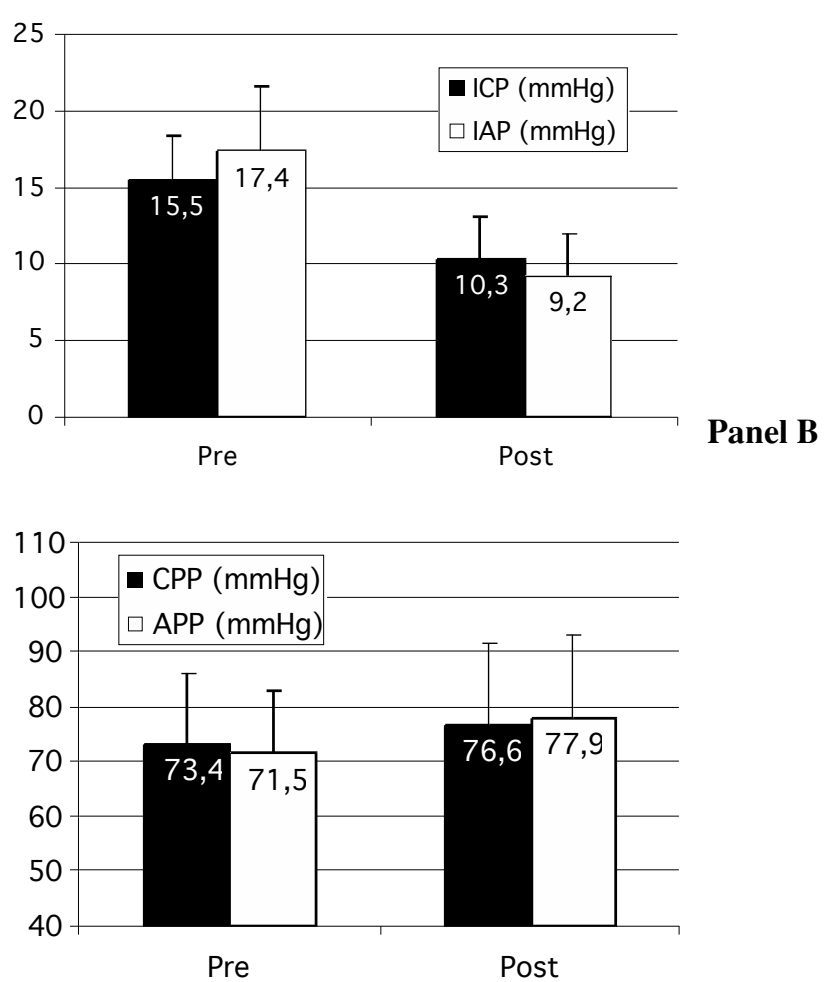

Figure 5: Bargraphs showing IAP and ICP (Panel $A$ ) and APP and CPP (Panel B) before (pre) and after (post) neuromuscular blockade (10mg cisatracurium) bolus administration (IAP: intraabdominal pressure, ICP: intracranial pressure, APP: abdominal perfusion pressure, CPP: cerebral perfusion pressure)

Modified from Deeren et al (20)

that are generally accepted to be safe. These results not only suggest that $\mathrm{ICH}$ in patients with $\mathrm{IAH}$ can be treated by administration of neuromuscular blockers, they also lend more support to the notion that there is a causal, rather than circumstantial, relationship between IAH and ICH. While neuromuscular blockers exert their effect on IAP through improved compliance of the abdominal wall, it is highly unlikely that they have any direct effect on ICP since the skull is a closed box lined by bony structures that are not responsive to neuromuscular blockers.

Some other treatment options have been described, such as continuously applied negative abdominal pressure or nonsurgical abdominal decompression (39). Both techniques will not be described here in detail. The findings of Deeren et al suggest that $\mathrm{ICH}$ can be treated in the presence of IAH by reducing IAH (20). The treatment of $\mathrm{IAH}$ as such lies beyond the scope of this text.
Preventive measures to avoid increase in ICP in patients who need to undergo abdominal surgery include the avoidance of induced pneumoperitoneum or if needed changing the type of insufflation gas $(27,48)$.

\section{CLINICAL RECOMMENDATIONS}

Naturally, all patients with intracranial hypertension need to be treated according to all accepted international standards and guidelines regarding $\mathrm{ICH}$ in their specific clinical situation. Although no randomized clinical trials have been conducted on any aspect of treatment of IAH, let alone the specific situation of ICH combined with IAH, we feel it is possible to make a few clinical recommendations based on the results of the previously mentioned animal and human studies.

- IAP monitoring is essential for all traumatic or nontraumatic patients at risk for $\mathrm{ICH}$ or $\mathrm{IAH}$ (according to the risk factors published by the World Society for the Abdominal Compartment Syndrome) (49)

- The presence of increased IAP can be an additional "extracranial" cause of intracranial hypertension in patients with abdominal trauma without overt craniocerebral lesions.

- In all patients with $\mathrm{ICH}$, preventive measures should be undertaken to avoid increase in IAP (the specific techniques involved lie outside the scope of this text)

- In all patients with IAH, a possible association with $\mathrm{ICH}$ should be considered and preventive measures should be taken (head of bed elevation, avoid hypervolemia, hypernatriemia and hyperthermia...)

- Neurologic status should be frequently monitored in patients with IAH

- Avoid hypervolemia in patients with IAH to prevent further increase in ICP. This may be difficult to achieve in a clinical setting, however, since volume management of these patients is complicated by the lack of reliable hemodynamic monitoring parameters. The use of routinely measured cardiac filling pressures is not reliable in the presence of IAH. This problem can be mediated by using (estimated) transmural filling pressures or by using volumetric hemodynamic monitoring parameters (6).

- Consider using APP (abdominal perfusion pressure; $A P P=M A P-I A P)$ as a resuscitation target in patients where ICP is not available and CPP can not be used as a target. 
- Provide adequate treatment for $\mathrm{IAH}$, especially if $\mathrm{ICH}$ is also present

- Avoid laparoscopy in patients at risk for $\mathrm{ICH}$. The pneumoperitoneum used for laparoscopy creates a situation analogous to experimental settings of $\mathrm{IAH}$ and $\mathrm{ICH}$ in which detrimental effects on ICP have been observed. This is especially important in trauma patients with associated brain and abdominal injuries.

\section{CONCLUSION}

A close relationship between IAP and ICP has been observed in several animal and human studies. The clinical impact of this association is dependent on the baseline ICP and the compensatory reserve of the patient. Some studies have reported good results in treating refractory $\mathrm{ICH}$ by abdominal decompression in patients with concomitant IAH. Clinicians should be aware of the different interactions between intra-abdominal, intrathoracic and intracranial pressures and be able to intervene adequately in the complex situation of combined intra-abdominal and intracranial hypertension. Monitoring of IAP and ICP in risk patients is essential.

In this review we summarized the current state of knowledge on this fascinating topic and formulated some clinical recommendations for management. It remains clear, however, that the complex phenomenon of IAH is still not completely understood and recommendations may change in the future as more clinical research is conducted.

\section{REFERENCES}

1. Malbrain ML, Chiumello D, Pelosi P, Bihari D, Innes R, Ranieri VM, et al. Incidence and prognosis of intraabdominal hypertension in a mixed population of critically ill patients: a multiple-center epidemiological study. Crit Care Med. 2005; 33: 315-22.

2. Malbrain ML, Chiumello D, Pelosi P, Wilmer A, Brienza N, Malcangi $V$, et al. Prevalence of intra-abdominal hypertension in critically ill patients: a multicentre epidemiological study. Intensive Care Med. 2004; 30: 822-9.

3. Ivatury RR, Cheatham ML, Malbrain ML, Sugrue M. Abdominal compartment syndrome. Georgetown: Landes Bioscience; 2006.

4. Malbrain ML. Is it wise not to think about intraabdominal hypertension in the ICU? Curr Opin Crit Care. 2004; 10: 132-45.

5. Sugrue M, Hallal A, D'Amours S. Intra-abdominal pressure hypertension and the kidney. In: Ivatury R, Cheatham M, Malbrain $M$, Sugrue $M$, editors. Abdominal compartment syndrome. Georgetown: Landes Bioscience; 2006; 119-28.
6. Cheatham M, Malbrain M. Cardiovascular implications of elevated intra-abdominal pressure. In: Ivatury $R$, Cheatham $M$, Malbrain $M$, Sugrue $M$, editors. Abdominal compartment syndrome. Georgetown: Landes Bioscience; 2006; 89-104.

7. Citerio G, Berra L. Central nervous system. In: Ivatury R, Cheatham M, Malbrain M, Sugrue M, editors. Abdominal compartment syndrome. Georgetown: Landes Bioscience; 2006; 144-56.

8. Bloomfield $\mathrm{GL}$, Ridings $\mathrm{PC}$, Blocher $\mathrm{CR}$, Marmarou A, Sugerman HJ. Effects of increased intra-abdominal pressure upon intracranial and cerebral perfusion pressure before and after volume expansion. J Trauma. 1996; 40: 936-41; discussion 41-3.

9. Bloomfield $\mathrm{GL}$, Ridings PC, Blocher CR, Marmarou A, Sugerman $\mathrm{HJ}$.A proposed relationship between increased intra-abdominal, intrathoracic, and intracranial pressure. Crit Care Med. 1997; 25: 496-503.

10. Joseph DK, Dutton RP, Aarabi B, Scalea TM. Decompressive laparotomy to treat intractable intracranial hypertension after traumatic brain injury. J Trauma. 2004; 57: 687-93; discussion 93-5.

11. Josephs LG, Este-McDonald JR, Birkett DH, Hirsch EF. Diagnostic laparoscopy increases intracranial pressure. J Trauma. 1994; 36 : 815-8; discussion 8-9.

12. Rosenthal RJ, Friedman RL, Kahn AM, Martz J, Thiagarajah S, Cohen $\mathrm{D}$, et al. Reasons for intracranial hypertension and hemodynamic instability during acute elevations of intra-abdominal pressure: observations in a large animal model. J Gastrointest Surg. 1998; 2: 415-25.

13. Rosenthal RJ, Hiatt JR, Phillips EH, Hewitt W, Demetriou AA, Grode M. Intracranial pressure. Effects of pneumoperitoneum in a large-animal model. Surg Endosc. 1997; 11: 376-80.

14. Citerio G, Andrews PJ. Intracranial pressure. Part two: Clinical applications and technology. Intensive Care Med. 2004; 30: 1882-5.

15. Citerio G, Vascotto E, Villa F, Celotti S, Pesenti A. Induced abdominal compartment syndrome increases intracranial pressure in neurotrauma patients: a prospective study. Crit Care Med. 2001; 29: 1466-71.

16. Halverson A, Buchanan R, Jacobs L, Shayani V, Hunt T, Riedel C, et al. Evaluation of mechanism of increased intracranial pressure with insufflation. Surg Endosc. 1998; 12: 266-9.

17. Halverson AL, Barrett WL, Iglesias AR, Lee WT, Garber SM, Sackier JM. Decreased cerebrospinal fluid absorption during abdominal insufflation. Surg Endosc. 1999; 13: 797-800.

18. Ben Haim M, Rosenthal RJ. Causes of arterial hypertension and splanchnic ischemia during acute elevations in intra-abdominal pressure with $\mathrm{CO} 2$ pneumoperitoneum: a complex central nervous system mediated response. Int J Colorectal Dis. 1999; 14: 227-36.

19. Ben-Haim M, Mandeli J, Friedman RL, Rosenthal RJ. Mechanisms of systemic hypertension during acute elevation of intraabdominal pressure. J Surg Res. 2000; 91: 101-5.

20. Deeren D, Dits H, Malbrain MLNG. Correlation between intraabdominal and intracranial pressure in nontraumatic brain injury. Intensive Care Med. 2005; 31: 1577-81.

21. Rosin D, Brasesco O, Varela J, Saber AA, You S, Rosenthal RJ, et al. Low-pressure laparoscopy may ameliorate intracranial hypertension and renal hypoperfusion. J Laparoendosc Adv Surg Tech A. 2002; 12: 15-9.

22. Rosin D, Rosenthal RJ. Adverse hemodynamic effects of intraabdominal pressure - is it all in the head? Int J Surg Investig. 2001; 2: 335-45.

23. Hanel F, Blobner M, Bogdanski R, Werner C. Effects of carbon dioxide pneumoperitoneum on cerebral hemodynamics in pigs. J Neurosurg Anesthesiol. 2001; 13: 222-6. 
24. Koehler RC, Chandra N, Guerci AD, Tsitlik J, Traystman RJ, Rogers $M C$, et al. Augmentation of cerebral perfusion by simultaneous chest compression and lung inflation with abdominal binding after cardiac arrest in dogs. Circulation. 1983; 67: 266-75.

25. Ratjen F, Trost A, Welker J, Spangenberg P, Wiesemann HG. The effect of rapid thoracoabdominal compressions on intracranial pressure in newborn lambs. Pediatr Res. 1995; 38: 664-7.

26. Palafox BA, Johnson MN, McEwen DK, Gazzaniga AB. ICP changes following application of the MAST suit. J Trauma. 1981; 21: 55-9.

27. Schob OM, Allen DC, Benzel E, Curet MJ,Adams MS, Baldwin NG, et al. A comparison of the pathophysiologic effects of carbon dioxide, nitrous oxide, and helium pneumoperitoneum on intracranial pressure. Am J Surg. 1996; 172: 248-53.

28. Irgau I, Koyfman Y, Tikellis JI. Elective intraoperative intracranial pressure monitoring during laparoscopic cholecystectomy. Arch Surg. 1995; 130: 1011-3.

29. Ertel W, Oberholzer A, Platz A, Stocker R, Trentz O. Incidence and clinical pattern of the abdominal compartment syndrome after "damage-control" laparotomy in 311 patients with severe abdominal and/or pelvic trauma. Crit Care Med. 2000; 28: 174753.

30. Bloomfield GL, Dalton JM, Sugerman HJ, Ridings PC, DeMaria EJ, Bullock R. Treatment of increasing intracranial pressure secondary to the acute abdominal compartment syndrome in a patient with combined abdominal and head trauma. J Trauma. 1995; 39: 1168-70.

31. Cooke SJ, Paterson-Brown S. Association between laparoscopic abdominal surgery and postoperative symptoms of raised intracranial pressure. Surg Endosc. 2001; 15: 723-5.

32. Ramos LA, Rifkinson N. Acute abdominal manifestations in patients with ventriculo-peritoneal shunts. Bol Asoc Med PR. 1990; 82: 541-3.

33. Miele VJ, Bendok B, Bloomfield SM, Ondra SL, Bailes JE. Ventriculoperitoneal shunt dysfunction in adults secondary to conditions causing a transient increase in intra-abdominal pressure: report of three cases. Neurosurgery. 2004; 55: 434.

34. Mirzayan MJ, Koenig K, Bastuerk M, Krauss JK. Coma due to meteorism and increased intra-abdominal pressure subsequent to ventriculoperitoneal shunt dysfunction. Lancet. 2006; 368: 2032.

35. Sugerman $H$, Windsor $A$, Bessos $M$, Wolfe L. Intra-abdominal pressure, sagittal abdominal diameter and obesity comorbidity. J Intern Med. 1997; 241: 71-9.
36. Sugerman $\mathrm{HJ}$. Effects of increased intra-abdominal pressure in severe obesity. Surg Clin North Am. 2001; 81: 1063-75, vi.

37. Sugerman HJ. Increased intra-abdominal pressure in obesity. Int J Obes Relat Metab Disord. 1998; 22: 1138.

38. Sugerman HJ, DeMaria EJ, Felton WL, III, Nakatsuka M, Sismanis A. Increased intra-abdominal pressure and cardiac filling pressures in obesity-associated pseudotumor cerebri. Neurology. 1997; 49: 507-11.

39. Saggi BH, Bloomfield GL, Sugerman HJ, Blocher CR, Hull JP, Marmarou AP, et al. Treatment of intracranial hypertension using nonsurgical abdominal decompression. J Trauma. 1999; 46: 646-51.

40. Sugerman H, Windsor A, Bessos M, Kellum J, Reines H, DeMaria E. Effects of surgically induced weight loss on urinary bladder pressure, sagittal abdominal diameter and obesity co-morbidity. Int J Obes Relat Metab Disord. 1998; 22: 230-5.

41. Sugerman HJ, Felton IW, III, Sismanis A, Saggi BH, Doty JM, Blocher $\mathrm{C}$, et al. Continuous negative abdominal pressure device to treat pseudotumor cerebri. Int J Obes Relat Metab Disord. 2001; 25: 486-90.

42. Kupersmith MJ, Gamell L, Turbin R, Peck V, Spiegel P, Wall M. Effects of weight loss on the course of idiopathic intracranial hypertension in women. Neurology. 1998; 50: 1094-8.

43. Sugerman HJ, Felton WL, 3rd, Sismanis A, Kellum JM, DeMaria EJ, Sugerman EL. Gastric surgery for pseudotumor cerebri associated with severe obesity. Ann Surg. 1999; 229: 634-40; discussion 40-2.

44. Giuseffi V, Wall M, Siegel PZ, Rojas PB. Symptoms and disease associations in idiopathic intracranial hypertension (pseudotumor cerebri): a case-control study. Neurology. 1991; 41: 23944.

45. Jennum P, Borgesen SE. Intracranial pressure and obstructive sleep apnea. Chest. 1989; 95: 279-83.

46. Binder DK, Horton JC, Lawton MT, McDermott MW. Idiopathic intracranial hypertension. Neurosurgery. 2004; 54: 538-51; discussion 51-2.

47. Lyons WS. Surgical abdominal decompression on relieving intractable ICH is of considerable general interest. J Trauma. 2004; 56: 723-4; author reply 4.

48. Holthausen UH, Nagelschmidt M, Troidl H. CO(2) pneumoperitoneum: what we know and what we need to know. World J Surg. 1999; 23: 794-800.

49. Malbrain ML, Cheatham ML, Kirkpatrick A, Sugrue M, Parr M, De Waele J, et al. Results from the International Conference of Experts on Intra-abdominal Hypertension and Abdominal Compartment Syndrome. I. Definitions. Intensive Care Med. 2006; 32: 1722-32. 